\title{
Effects of Triptolide on Cell Viability, Secretion of Inflammatory Cytokines, and Gene Expression of Severe Coronavirus Disease 2019凶COVID-19ð Pseudovirus Cell Model
}

\section{junyong han}

Fujian Academy of Medical Sciences

\section{Huiqing Que}

Fujian Academy of Medical Sciences

Wei Li

Fujian Academy of Medical Sciences

Shijie Xue

Fujian Academy of Medical Sciences

Sui Lin

Fujian Academy of Medical Sciences

gang chen ( $\nabla$ chengangfj@163.com )

Fujian Provincial Hospital https://orcid.org/0000-0002-8105-2384

\section{Research}

Keywords: Triptolide, COVID-19, Transcriptome, Cell viability, Gene expression.

Posted Date: October 12th, 2021

DOI: https://doi.org/10.21203/rs.3.rs-942032/v1

License: (c) (i) This work is licensed under a Creative Commons Attribution 4.0 International License. Read Full License 


\section{Abstract}

Background: Acute respiratory distress syndrome (ARDS), which is caused by severe immune cell response and associated alveolar inflammation, is also a critical complication in hospitalized patients with COVID-19. Triptolide is a powerful anti-inflammatory and immunosuppressive drug and is proved to help relieve the inflammation of ARDS. However, its anti-inflammatory effect on COVID-19 patients with ARDS complications remains uncertain.

Methods: In this study, human normal lung epithelial cells (BEAS-2B), the pseudovirus system of syndrome coronavirus 2(SARS-CoV-2) and lipopolysaccharide (LPS) were used to construct as severe COVID-19-pseudovirus cell model to explore the effects of triptolide on cell viability, secretion of inflammatory cytokines, and gene expression.

Results: The results showed that triptolide increased cell viability, decreased the secretion levels of cytokines IL-6, TNF-a, and increased the expression of IL-10. Furthermore, transcriptome analysis in this cell models showed that the Differentially expressed genes (DEGs) were related to plasma membrane integrity, metabolic activity and mitochondrial function, and were associated with TNF, FOXO, mTOR and MAPK signaling pathways.

Conclusion: Take into consideration previous studies on the functions of triptolide in BEAS-2B cells, the current study indicated that triptolide can play a critical role in protecting against inflammatory damage and maintaining the normal physiological function of BEAS-2B cells in response to pseudovirus and LPS infection.

\section{Background}

COVID-19 is a clinical syndrome caused by infection with severe acute respiratory SARS-CoV-2. Although most SARS-CoV-2-infected individuals experience mild symptoms, some patients suffer from severe complications like ARDS and systemic inflammation [1-2]. Triptolide is an epoxy diterpene lactone compound extracted from the roots, leaves, flowers and fruits of Tripterygium wilfordii, and it manifests multiple biological effects, including anti-cancer [3], anti-inflammation [4-6] and immunosuppression [7]. The results from the present study suggest that the anti-inflammatory effect of triptolide against LPS-induced ARDS may attribute to its ability to inhibit the secretion of tumour necrosis factor-a(TNF-a), interleukin-1 $\beta(\mathrm{IL}-1 \beta)$ and interleukin-6(IL-6) [8-10].

However, the anti-inflammatory function of triptolide in patients with severe COVID-19 accompanied by ARDS and systemic inflammation is less understood. With the help of Connectivity Map (CMap) database [11] and network pharmacology and data mining [12], recent research indicates that triptolide would provide new options for improving treatment of patients infected with SARS-CoV-2. In addition, triptolide may serve as an important function in promoting autophagy and inhibiting apoptosis [13], reducing oxidative stress [14-15] and improving antioxidant activity [16], all of which were correlated in terms of the protective effect of cell. 
To get a better understanding of its functions in severe COVID-19, we explored the effects of triptolide on cell viability, secretion of inflammatory cytokines, and gene expression in severe COVID-19-pseudovirus cell model. It was observed that triptolide moderately increased cell viability in BEAS-2B and inhibited the secretion of inflammatory cytokines. More importantly, the DEGs provided convincing evidence showing that triptolide maintains normal physiological functions of the severe COVID-19-pseudovirus cell model through TNF, FOXO, mTOR and MAPK signaling pathway.

\section{Materials And Methods}

\section{Cells and Drugs}

Human normal lung epithelial cell line BEAS-2B was purchased from Shanghai Cell Bank. BEAS-2B cells were cultured in specific medium for BEAS-2B cells (CM-0496(ProCell)), with 10\% fetal bovine serum (FBS) and $5 \% \mathrm{CO} 2$ at $37^{\circ} \mathrm{C}$. The triptolide (Provided by Fujian Academy of Medical Sciences) was dissolved in $0.1 \%$ dimethyl sulfoxide (DMSO, DMSO had little effect on subsequent experiments, so the data of DMSO in the experiment were not listed, and the final concentration of DMSO in the medium was adjusted to less than $0.1 \%$ ). The concentrations of triptolide $L, M$ and $H$ were $2.5 \mathrm{ng} / \mathrm{ml}, 5 \mathrm{ng} / \mathrm{mL}$ and $10 \mathrm{ng} / \mathrm{mL}$, respectively.

\section{Building the severe COVID-19-pseudovirus cell model}

The pseudovirus system was purchased from Wuhan Shumi Brain Science and Technology Co., Ltd., which included PV-SARS-CoV-2-S-VSV- $\triangle$ g-EGFP (V04001,1.30E+07IFU/mL); RLV-CMV-SARS-CoV-2-SMcHerry-WPRE(LV-0614,1.00e+06TU/mL). RLV-CMV-ACE2-PGK-PURO-WPRE (LV-0707,1.00E+06 TU/mL), and the infection complex number (MOI) is 1. Referring to previous studies [17], cells were inoculated with 6-well plates at $1 \times 10^{6}$, and the complete medium was replaced 24 hours later. Pseudoviral system was firstly added according to $\mathrm{MOI}=1$, and then the virus supernatant was removed at 6 hours, and the fresh complete medium was replaced in the meantime. The experimental group was added with $100 \mu \mathrm{g} / \mathrm{mL}$ LPS (Sigma) for $24 \mathrm{~h}$, and the intervention time of triptolide was $72 \mathrm{~h}$.

\section{Cell Viability Assay}

Take BEAS-2B cells $\left(1 \times 10^{4}\right.$ cells $/$ well) and add $2.5 \mathrm{ng} / \mathrm{mL}, 5 \mathrm{ng} / \mathrm{mL}, 10 \mathrm{ng} / \mathrm{mL}$ of triptolide, respectively. The effects of triptolide on the cell viability of BEAS-2B at 24, 48 and 72 hours were analyzed with the VI-CELL XRVI-CELL (Beckman Coulter). Data are expressed as mean standard deviations with three duplicates, and the results are tested by analysis of variance and Duncan multipolar test (Pख0.05).

\section{Measurement of cytokines}

Cell supernatant was collected and centrifugated at $3500 \mathrm{rpm}$ for $10 \mathrm{mins}$. Levels of IL-6, IL-10 and TNF-a in the supernatant were analyzed using ELISA kits, and optical density (OD) values were determined using a multifunctional enzyme plate analyzer (Synergy 2, USA, Bio-Tek, Inc.). 


\section{RNA extraction and detection}

Total RNA was extracted by Trizol (Beijing Tiangen Biochemical Co., Ltd.), and then RNA quality was detected. The purity of RNA was detected by Nanodrop spectrophotometer (Implen, CA, USA). Agilent 2100 (Agilent Technologies, CA, USA) assesses RNA integrity.

\section{Differential expression analysis}

Deseq (1.10.1) was used for differential expression analysis. The P-values of the results of differential expression analysis were controlled for false discovery rate (FDR) with Benjamini and Hochberg methods. The standard of differential gene screening is generally $Q<0.05$.

Gene Ontology(GO) enrichment analysis

GO enrichment analysis of the DEGs was implemented by the GOseq R packages based on Wallenius non-central hyper-geometric distribution [18], which can adjust for gene length bias in DEGs.

Kyoto Encyclopedia of Genes and Genomes (KEGG) pathway enrichment analysis

KEGG [199]is a database resource for understanding high-level functions and utilities of the biological system, such as the cell, the organism and the ecosystem, from the perspective of molecular level information, especially large-scale molecular datasets generated by genome sequencing and other highthroughput experimental technologies. We used KOBAS [20] software to test the statistical enrichment of differential expression genes in KEGG pathways.

\section{Statistical Analysis}

GraphPad Prism 8 was used to perform data analysis. Data for the cell viability assay were calculated from three independent experiments with each experiment containing six replicates. Results were presented as mean \pm SD. Data comparison was performed using the analysis of variance (ANOVA, oneway or two-way), followed by Dunnett's or Tukey's post hoc tests. Results were considered statistically significant $(P<0.05)$.

\section{Results}

\section{Effect of triptolide on Cell Viability and inflammatory cytokines secretion}

As is shown in Fig.S1A-C, the concentration of triptolide less than $10 \mathrm{ng} / \mathrm{ml}$ had no significant cytotoxic effects on the BEAS-2B. Compared with the model group (Virus + LPS), the cell viability of BEAS-2B was improved after $72 \mathrm{~h}$ of triptolide treatment $(P<0.05)$ (Fig. 1A-C). In addition, Fig. 1D-F shows that triptolide decrease the levels of cytokines IL-6, TNF-a and increase the levels of IL-10 $(P<0.01)$. These results verified that triptolide can help reduce the inflammatory response and improve cell survival activity in severe COVID-19-pseudovirus cell model. 


\section{Effect of triptolide on Gene Expression in severe COVID-19-pseudovirus cell model.}

The gene expression affected by triptolide treatment was detected by transcriptomics. DEGs were highlighted in green and red in the volcano plots (Fig. 2A). As Fig. 2C shows, 639 DEGs in model control group, and 1,236 DEGs in triptolide intervention group, were identified respectively. Moreover, the differential gene expression cluster analysis showed that the expression patterns of genes in the similar color cluster region were identical, indicating that these genes may have similar functions or participate in the regulation of the same metabolic pathway (Fig. 2B). The top 30 up- and downregulated DEGs are summarized in Table.S1.

\section{Go Enrichment Analysis}

A number of $\mathrm{GO}$ terms belonging to biological process (cellular metabolic process, nucleic acid metabolic process, localization, transport and oxidation-reduction process), cellular component (intracellular, organelle, cytoplasm, membrane-bounded organelle and endoplasmic reticulum), molecular function (nucleic acid binding, protein binding and oxidoreductase activity) were significantly enriched within the dataset (Fig. 3A-B).By regulating these genes, triptolide can affect cellular activity in terms of metabolic activity and plasma membrane integrity.

\section{Kegg Enrichment Analysis}

KEGG enrichment analysis provided additional information on possible functional pathways that the DEGs are involved in (Fig. 4A-B).Further bioinformatics analysis showed that the downregulated DEGs were significantly enriched in signaling pathways such as the TNF, FOXO, mTOR and MAPK; metabolic pathways and cellular physiological processes such as mitophagy, autophagy and endocytosis (Fig. 4A).For the upregulated DEGs, the enriched pathways were mainly involved in ribosome, oxidative phosphorylation, lysosome, ECM-receptor interaction and glycolysis/ gluconeogenesis (Fig. 4B).

\section{Discussion}

In the current study, we observe the protection effects of triptolide on cell viability of severe COVID-19pseudovirus cell model, and we found that triptolide inhibits the secretion of inflammatory cytokine IL-6 and TNF-a, and enhances the secretion of anti-inflammatory cytokine IL-10. What's more, to better understand the mechanisms underlying regulation of cell viability, multiple signaling pathways heavily involved and well-studied, including TNF, FOXO, mTOR and MAPK, mitophagy, autophagy and metabolic pathways have now been shown to be associated with triptolide.

Our observation was in line with previous studies that triptolide protects cell viability of neural cells [2122], cardiac cells [23] and the neuroinflammation response induced by LPS [24]. However, these previous studies were mainly carried out with NF-kappa B or any other single signaling pathway. There are also 
many reports on the role of triptolide in antiviral mechanisms. For example, triptolide inhibits human immunodeficiency virus type 1 replication [25], and triptolide impaired the number of viral DNA copies and virion production [26]. Whereas, whether triptolide can also protect the activity of cells infected with SARS-CoV-2 and exert its powerful anti-inflammatory effect has not been reported when our research was carried out. Besides, mitochondria are mainly related to cell bioenergy, which is an important cause of cell apoptosis and death [27], and mitochondria also impact on complex cellular processes including in the regulation of autophagy [28-29], immune response [30] and some other complex cell process. Our study found that the levels of mitochondria-related genes were regulated by triptolide (Fig. 4A, Table. 1 and Fig.S2), indicating that the improvement of mitochondrial function was an important factor in the protection of cell activity of triptolide.

Existing studies have confirmed that triptolide plays an anti-inflammatory role in different diseases involving different signaling pathways. For example, triptolide induces protective autophagy in human cervical cancer cells by targeting phosphoinositol 3-kinase/Akt/mTOR, p38, MAPK, p53 and FOXO3a signaling pathways [31]. And in angiocardiopathy, ErbB, Hippo and hypoxia-inducible factor-1a (HIF-1a) signaling pathways are involved [31-33]. In addition, FOXO and MAPK pathways are involved in a variety of cellular functions and have clinical significance, including its effects on cell cycle arrest, cell differentiation, cell proliferation, migration, senescence and apoptosis [34-35]. These signaling pathways's functions have also been confirmed in this study, and our research focuses on the TNF, FOXO, mTOR and MAPK signaling pathways and explores the effects of triptolide on cell viability and antiinflammatory effect.

In view of the fact that monitoring cell viability is a key task for basic research, such as apoptosis, necrosis and drug discovery, this study focused on maintaining cell activity with triptolide. Through our analysis, in addition to regulating the expression level of inflammatory factors, it was found that triptolide can change the expression of cell viability-related genes and proteins through a variety of signaling pathways, so as to protect cell activity by down-regulating the expression levels of mitochondrial damage, autophagy and other related genes. Taking all these factors together, triptolide can maintain the normal physiological function of BEAS-2B cells by regulating the integrity of plasma membrane, protecting mitochondrial function and balancing normal cell metabolic activity, and thus protect against pseudovirus and LPS-mediated BEAS-2B cells damage.

On the other hand, it is worth noting that large doses or long-term use of triptolide also can cause many adverse reactions, including membrane damage, mitochondrial disruption, metabolism dysfunction, oxidative stress, apoptosis and autophagy, so there is also a concern between the efficacy and toxicology of triptolide. Of course, TNF, FOXO, mTOR and MAPK signaling pathway are not independent of each other in the action of triptolide, and these signaling pathways are interrelated. Although we have selected a certain amount of related genes and signaling pathways, many of the targets and exact mechanisms involved in these events remain unknown. Therefore, the exact mechanism and function of triptolide in severe COVID-19-pseudovirus cell model is also worth further study. 


\section{Conclusions}

In this study, transcriptomics were used to analyze the mechanism of triptolide enhancing cell viability in severe COVID-19 pseudovirus model. In addition, this study provides reference for the clinical application of triptolide in alleviating ARDS symptoms of severe COVID-19 patients.

\section{Abbreviations}

COVID-19

Coronavirus Disease 2019

ARDS

Acute respiratory distress syndrome

SARS-CoV-2

syndrome coronavirus 2

LPS

lipopolysaccharide

DEGs

Differentially expressed genes

CMap

Connectivity Map

TNF-a

Tumour Necrosis Factor-a

IL-1 $\beta$

interleukin-1 $\beta$

IL-6

interleukin-6

FBS

fetal bovine serum

DMSO

dimethyl sulfoxide

OD

optical density

GO

Gene Ontology

KEGG

Kyoto Encyclopedia of Genes and Genomes.

\section{Declarations}

Ethics approval and consent to participate 
Not applicable.

\section{Consent for publication}

Not applicable.

\section{Availability of data and material}

All datasets and reagents are available from the corresponding author on reasonable request.

\section{Funding}

This work was supported by the High-Level Hospital grants from Fujian Provincial Hospital (No.2017LHJJ06), and Major Health Scientific Research Project of Fujian Province (No.2021ZD02002).

\section{Competing interests}

The authors declare no conflicts of interest.

\section{Authors' contributions:}

JYH developed cell models, performed experiments and data analysis, contributed to manuscript writing.HQX and WL performed experiments and data analysis. SJX and SL supervised the research and interpretation of data. GC conceived the primary hypothesis, designed the research, analyzed and interpreted the data. All authors read and approved the final manuscript.

\section{Acknowledgments}

The authors thank Professor Yi Chen (Fujian Academy of Medical Sciences, Fuzhou, China) for reviewing the article.

\section{References}

1. Bonaventura Aldo, Vecchié Alessandra, Dagna Lorenzo et al. Endothelial dysfunction and immunothrombosis as key pathogenic mechanisms in COVID-19.[J] .Nat Rev Immunol, 2021, 21: 319-329.

2. Lee Jeong Seok, Park Seongwan, Jeong Hye Won et al. Immunophenotyping of COVID-19 and influenza highlights the role of type I interferons in development of severe COVID-19.[J] .Sci Immunol, 2020; doi:10.1126/sciimmunol.abd1554.

3. Liang Xia, Xie Renxiang, Su Jinfeng et al. Inhibition of RNA polymerase III transcription by Triptolide attenuates colorectal tumorigenesis.[J] .J Exp Clin Cancer Res, 2019, 38: 217.

4. Wei Dong, Huang Zhihong, Anti-inflammatory effects of triptolide in LPS-induced acute lung injury in mice.[J] .Inflammation, 2014, 37: 1307-16. 
5. Gao Jianling, Zhan Ying, Chen Jun et al. Triptolide ameliorates lipopolysaccharide-induced acute lung injury in rats.[J] .Eur J Med Res, 2013, 18: 58.

6. Yang Yi-Qi, Yan Xiao-Teng, Wang Kai et al. Triptriolide Alleviates Lipopolysaccharide-Induced Liver Injury by Nrf2 and NF-kB Signaling Pathways.[J] .Front Pharmacol, 2018, 9: 999.

7. Gu Chuan, Liu Fei, Luo Xusong et al. Triptolide Reduces the Required Dose of Tacrolimus by Attenuating Inflammation, Enhancing Immunosuppression, and Increasing Donor Chimerism in a Heterotopic Hindlimb Transplantation Model.[J] .Plast Reconstr Surg, 2016, 138: 1243-1253.

8. Wang Xian, Zhang Lei, Duan Wei et al. Anti-inflammatory effects of triptolide by inhibiting the NF-KB signalling pathway in LPS-induced acute lung injury in a murine model.[J] .Mol Med Rep, 2014, 10: 447-52.

9. Yang Huilin, Qian Hong, Liu Bo et al. Triptolide dose-dependently improves LPS-induced alveolar hypercoagulation and fibrinolysis inhibition through NF-KB inactivation in ARDS mice.[J] .Biomed Pharmacother, 2021, 139: 111569.

10. Chen Jun, Gao Jianlin, Yang Jianping et al. Effect of triptolide on the regulation of ATP-binding cassette transporter A1 expression in lipopolysaccharide-induced acute lung injury of rats.[J] .Mol Med Rep, 2014, 10: 3015-20.

11. Chen WY, Fang ZX, Lv XD et al. Prediction of potential therapeutic drugs against SARS-CoV-2 by using Connectivity Map based on transcriptome data.[J] .Eur Rev Med Pharmacol Sci, 2021; doi:10.26355/eurrev_202104_25567.

12. Yu Mei-Xiang, Song Xin, Ma Xiao-Qin et al. Investigation into molecular mechanisms and highfrequency core TCM for pulmonary fibrosis secondary to COVID-19 based on network pharmacology and data mining.[J] .Ann Palliat Med, 2021, 10: 3960-3975.

13. Zhu Ning, Ruan Jianwei, Yang Xinming et al. Triptolide improves spinal cord injury by promoting autophagy and inhibiting apoptosis.[J] .Cell Biol Int, 2020, 44: 785-794.

14. Xu Pengjuan, Wang Hui, Li Zhigui et al. Triptolide attenuated injury via inhibiting oxidative stress in Amyloid-Beta25-35-treated differentiated PC12 cells.[J] .Life Sci, 2016, 145: 19-26.

15. Han Fengmei, Zhang Jingyi, Li Kun et al. Triptolide protects human retinal pigment epithelial ARPE19 cells against high glucose-induced cell injury by regulation of miR-29b/PTEN.[J] .Arch Physiol Biochem, 2020; doi:10.1080/13813455.2020.1797101.

16. Zhang Jianlin, Guan Yongmei, He Liangfei et al. Influence of a combination of triptolide and ferulic acid on the activities of CYP450 enzymes and oxidative stress in HaCaT cells.[J] .Exp Ther Med, 2020, 20: 157.

17. Nie J, Li Q, Wu J, et al. Establishment and validation of a pseudovirus neutralization assay for SARSCoV-2[J]. Emerging Microbes \& Infections.2020,9:680-686.

18. Young Matthew D, Wakefield Matthew J, Smyth Gordon K et al. Gene ontology analysis for RNA-seq: accounting for selection bias.[J].Genome Biol, 2010, 11: R14.

19. Kanehisa Minoru, Araki Michihiro, Goto Susumu et al. KEGG for linking genomes to life and the environment.[J] .Nucleic Acids Res, 2008; doi:10.1093/nar/gkm882. 
20. Mao Xizeng, Cai Tao, Olyarchuk John G et al. Automated genome annotation and pathway identification using the KEGG Orthology (KO) as a controlled vocabulary.[J] .Bioinformatics, 2005, 21: 3787-93.

21. Wang J, Shi ZQ, Xu X et al. Triptolide inhibits amyloid- $\beta$ production and protects neural cells by inhibiting CXCR2 activity.[J] .J Alzheimers Dis, 2013, 33: 217-29.

22. Zhang Bin, Song Cunfeng, Feng Bo et al. Neuroprotection by triptolide against cerebral ischemia/reperfusion injury through the inhibition of NF-KB/PUMA signal in rats.[J] .Ther Clin Risk Manag, 2016; doi:10.2147/TCRM.S106012.

23. Wen He-Ling, Liang Zhong-Shu, Zhang Rui et al. Anti-inflammatory effects of triptolide improve left ventricular function in a rat model of diabetic cardiomyopathy.[J] .Cardiovasc Diabetol, 2013, 12: 50.

24. Dai Yu-Qiao, Jin Dao-Zhong, Zhu Xing-Zu et al. Triptolide inhibits COX-2 expression via NF-kappa B pathway in astrocytes.[J] .Neurosci Res, 2006, 55: 154-60.

25. Wan Zhitao, Chen Xulin, Triptolide inhibits human immunodeficiency virus type 1 replication by promoting proteasomal degradation of Tat protein.[J] .Retrovirology, 2014, 11: 88.

26. Long Cong, Guo Wei, Zhou Heng et al. Triptolide decreases expression of latency-associated nuclear antigen 1 and reduces viral titers in Kaposi's sarcoma-associated and herpesvirus-related primary effusion lymphoma cells. [J]. Int J Oncol, 2016, 48: 1519-30.

27. Liu X, Kim C N, Yang J et al. Induction of apoptotic program in cell-free extracts: requirement for dATP and cytochrome c.[J] .Cell, 1996, 86: 147-57.

28. Gomes Ligia C, Di Benedetto Giulietta, Scorrano Luca, During autophagy mitochondria elongate, are spared from degradation and sustain cell viability.[J] .Nat Cell Biol, 2011, 13: 589-98.

29. Rambold Angelika S, Kostelecky Brenda, Elia Natalie et al. Tubular network formation protects mitochondria from autophagosomal degradation during nutrient starvation.[J] .Proc Natl Acad Sci U S A, 2011, 108: 10190-5.

30. Yasukawa Kai, Oshiumi Hiroyuki, Takeda Makoto et al. Mitofusin 2 inhibits mitochondrial antiviral signaling.[J] .Sci Signal, 2009; doi:10.1126/scisignal.2000287.

31. Qin Guangyi, Li Ping, Xue Zhuowei, Triptolide induces protective autophagy and apoptosis in human cervical cancer cells by downregulating Akt/mTOR activation.[J] .Oncol Lett, 2018, 16: 3929-3934.

32. MicroRNA expression, targeting, release dynamics and early-warning biomarkers in acute cardiotoxicity induced by triptolide in rats.[J] .Biomed Pharmacother, 2019, 111: 1467-1477.

33. Li Xugui, Lu Qilin, Xie Wei et al. Anti-tumor effects of triptolide on angiogenesis and cell apoptosis in osteosarcoma cells by inducing autophagy via repressing Wnt/ $\beta$-Catenin signaling. [J] .Biochem Biophys Res Commun, 2018, 496: 443-449.

34. Sun Yu, Liu Wen-Zhou, Liu Tao et al. Signaling pathway of MAPK/ERK in cell proliferation, differentiation, migration, senescence and apoptosis.[J] .J Recept Signal Transduct Res, 2015, 35: 600-4. 
35. Tia Neelam, Singh Alok Kumar, Pandey Poorti et al. Role of Forkhead Box O (FOXO) transcription factor in aging and diseases.[J] .Gene, 2018, 648: 97-105.

\section{Tables}

Table 1 is not available with this version.

\section{Figures}

Fig1
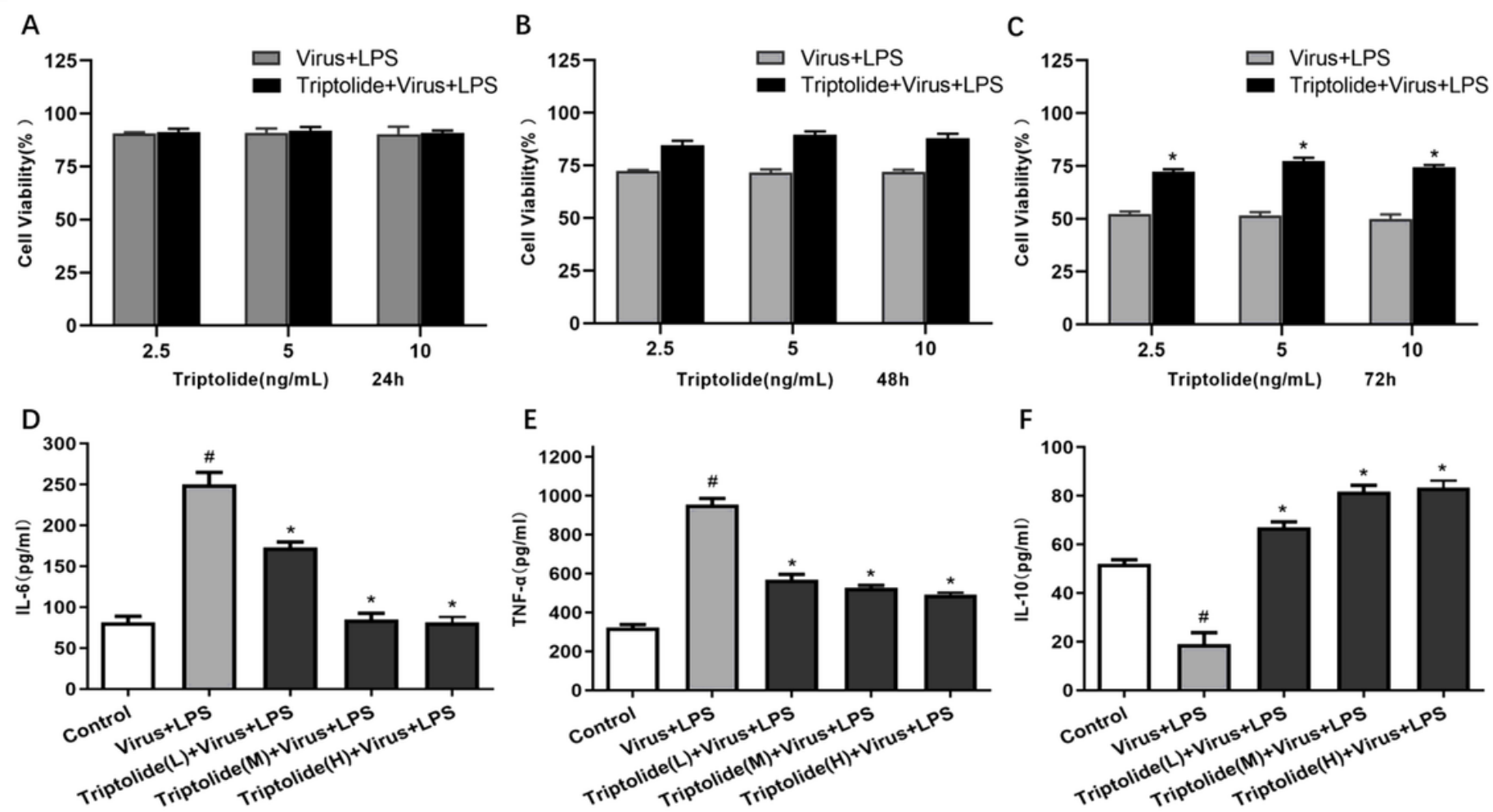

Figure 1

Effect of triptolide on Cell Viability and inflammatory cytokines secretion. (A-C) The effects of triptolide on severe COVID-19-pseudovirus cell viability for 24, 48 and 72h. (D-F) The cytokines of IL-6, TNF-a and IL-10 were detected by ELISA. Data are shown as mean \pm SEM; \#P $<0.01$ vs. control group; $* p<0.05$ vs. Virus + LPS group. 
Fig2

A

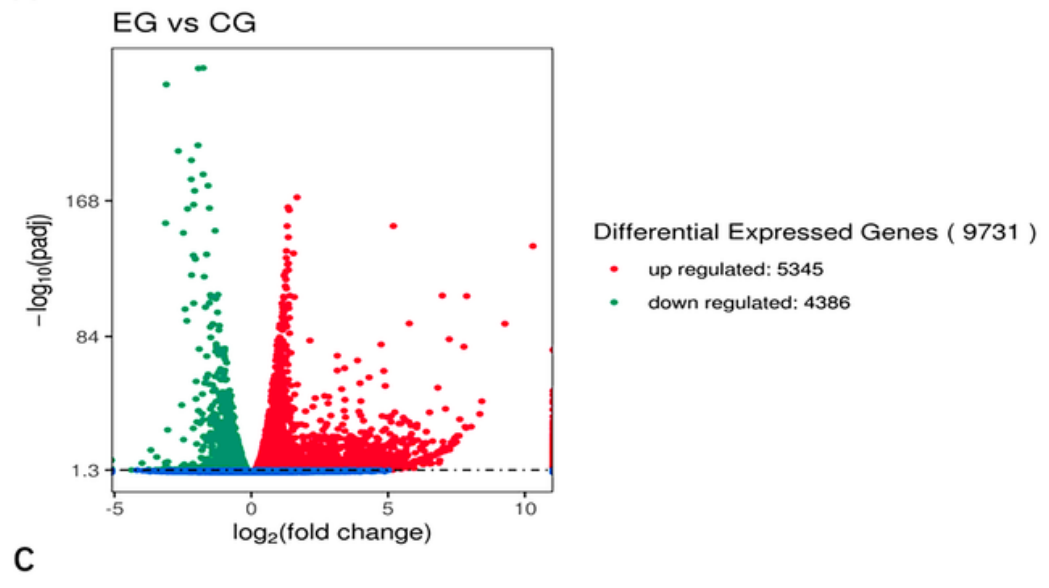

EG

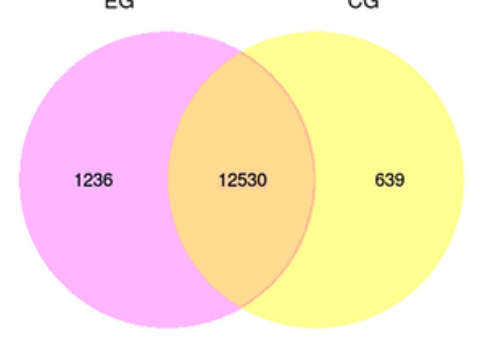

B

Cluster analysis of differentially expressed genes

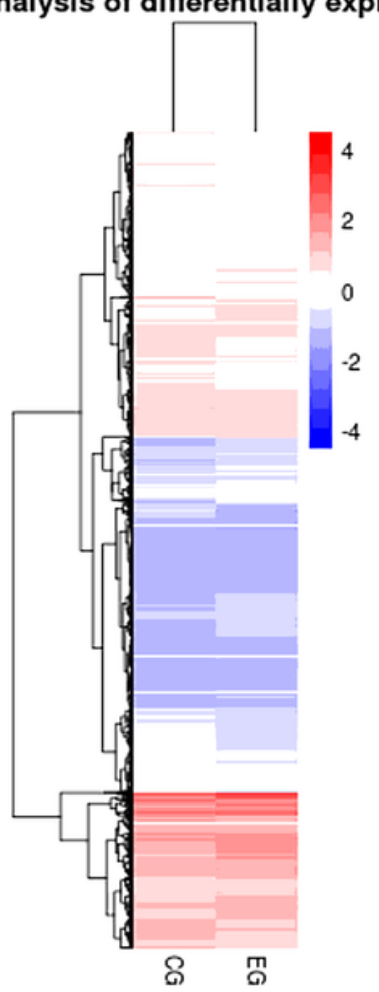

Figure 2

Effect of triptolide on Gene Expression. (A) The volcano map shows the overall distribution of different genes. (B) Clustering Analysis of Differential Gene Expression: hierarchical clustering analysis is conducted with the expression levels of different genes under different experimental conditions (using Log10 (FPKM+1) value). Regions with different colors represent different cluster grouping information. (C)Venn diagram of differentially expressed genes. (EG: Triptolide was used in the severe cell model group of coronavirus; CG: Coronavirus severe cell model group). 
Fig3

A

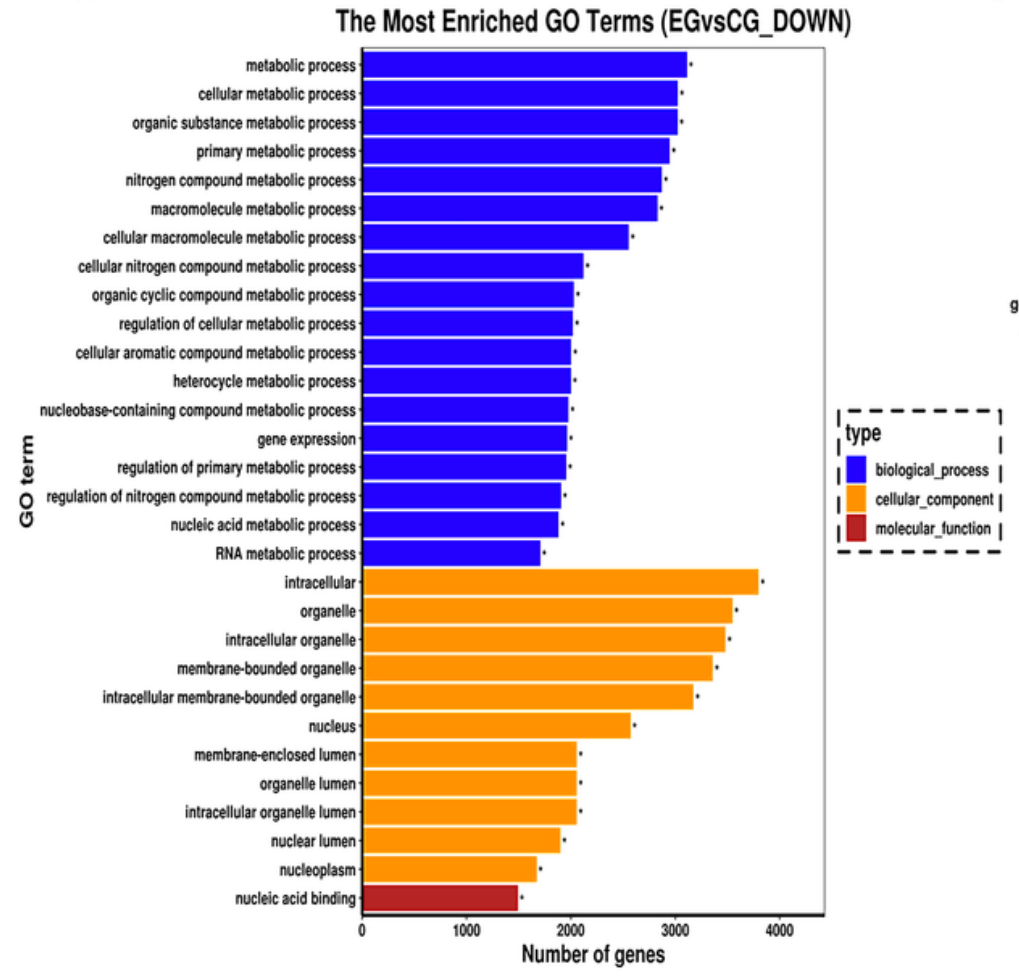

B

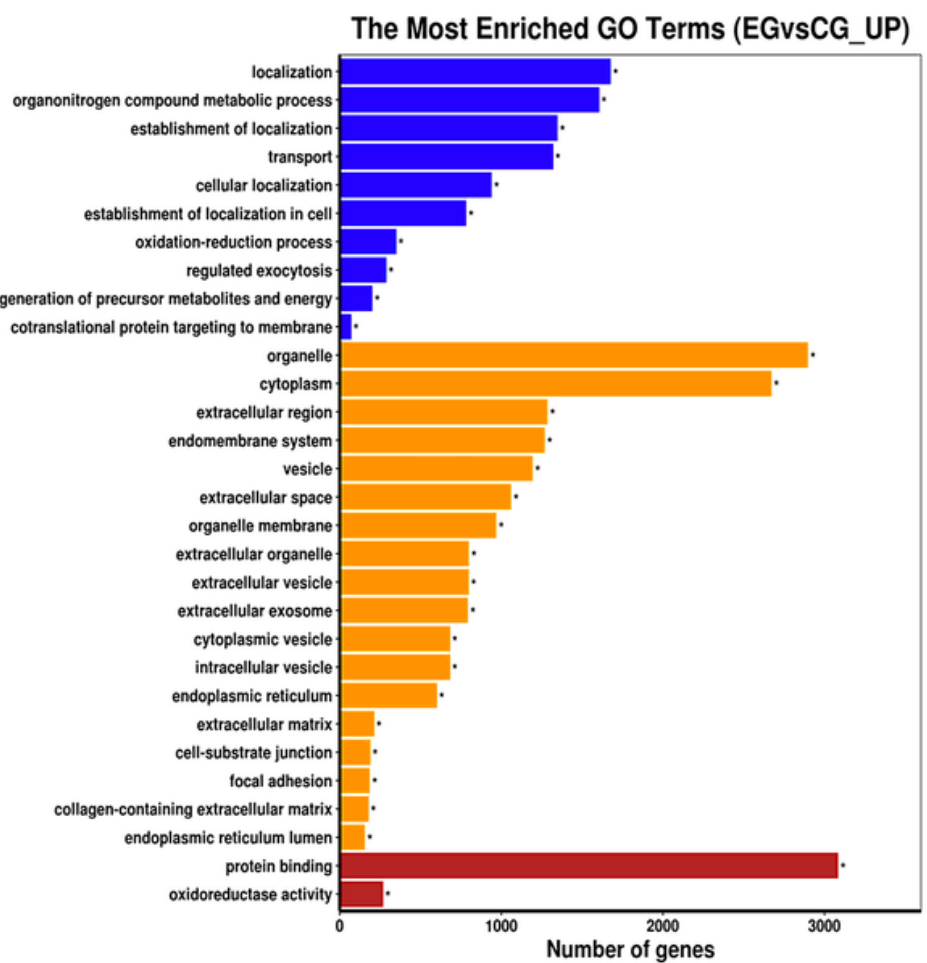

\section{Figure 3}

Gene Ontology (GO) Analysis. (A) down-regulated GO enrichment analysis of EG compared with CG group; (B)GO enrichment analysis of EG up-regulated compared with CG group; (The ordinate is the enriched GO term, and the abscissa is the number of differentially expressed genes in the term. Different colors are used to distinguish biological processes, cell components and molecular functions, and " $\star$ " is the significantly enriched GO term). 


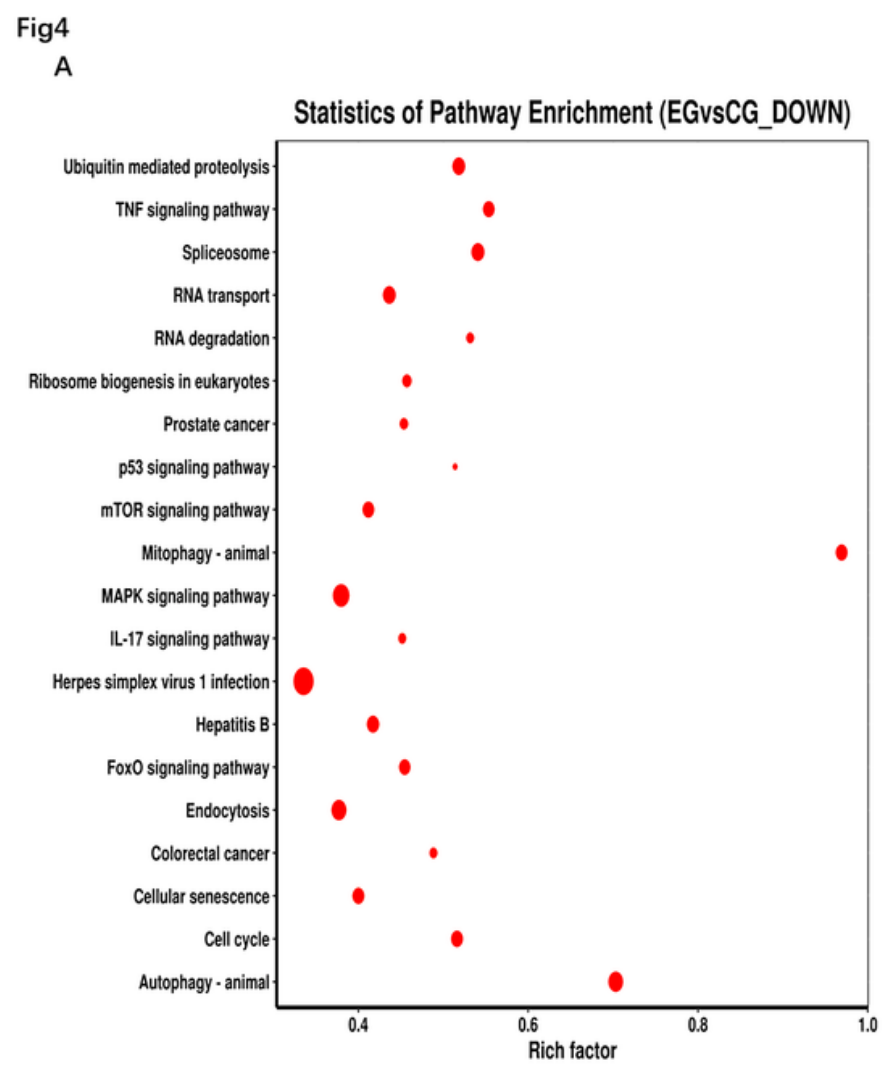

B

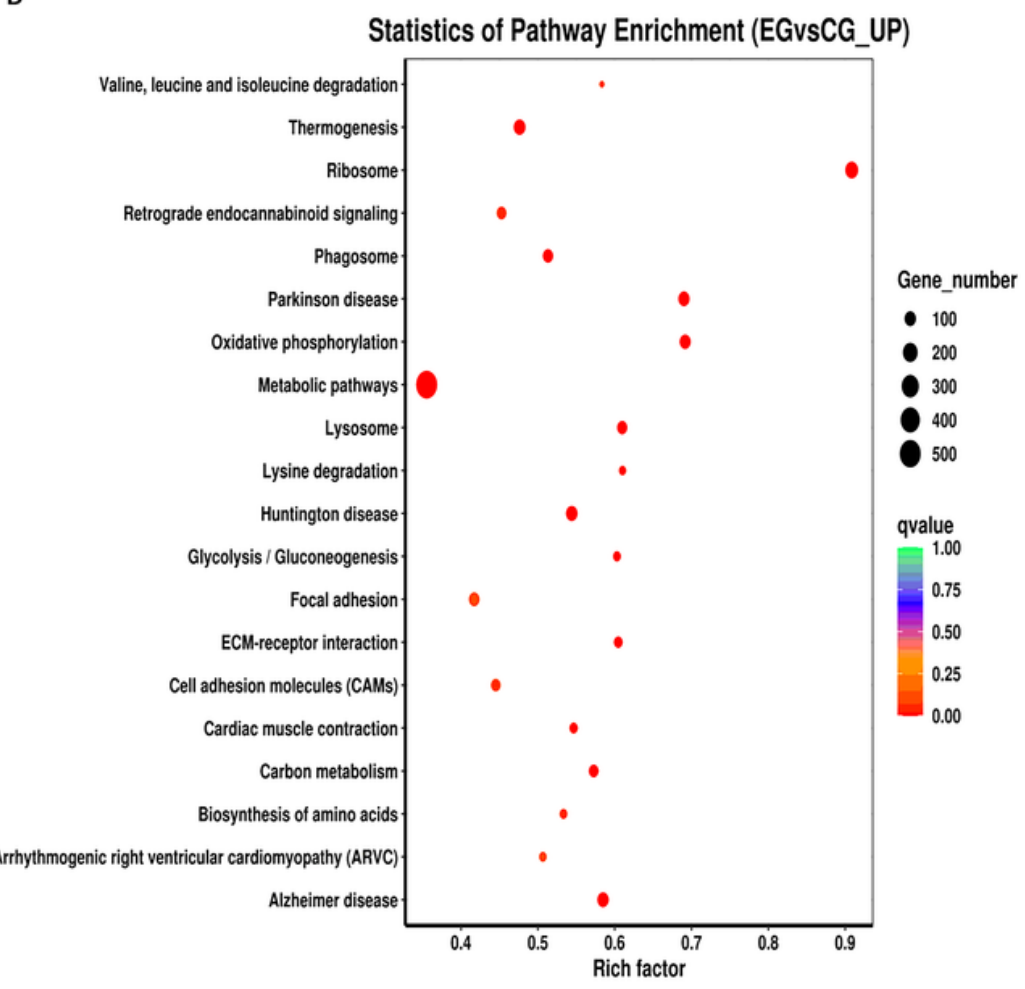

Figure 4

Kyoto Encyclopedia of Genes and Genomes (KEGG) enrichment analysis. (A-B) shows 20 pathways with the most significant enrichment in EG compared to CG group, which are up-regulated and down-regulated. (Rich factor refers to the ratio of the number of differentially expressed genes enriched in Pathway to the number of annotated genes. The higher the Rich Factor value is, the greater the enrichment degree is. Qvalue (value range 0-1) is the Pvalue corrected through multiple hypothesis testing. The closer the Qvalue is to zero, the more significant the enrichment is).

\section{Supplementary Files}

This is a list of supplementary files associated with this preprint. Click to download.

- S1.tif

- S2.tif

- Table.S1.tif 